\title{
Characterization of an iron-inducible Haemaphysalis longicornis tick-derived promoter in an Ixodes scapularis-derived tick cell line (ISE6)
}

\author{
Emmanuel Pacia Hernandez ${ }^{1,2}$, Kodai Kusakisako ${ }^{1,3}$, Takeshi Hatta ${ }^{4}$ and Tetsuya Tanaka ${ }^{1,2^{*}}$ (D)
}

\begin{abstract}
Background: Ticks are important vectors of disease-causing pathogens. With the rise of resistance to chemical acaricides, alternative methods in tick control are warranted. Gene manipulation has been successful in controlling mosquitoes and mosquito-borne diseases and is now looked upon as a candidate method to control ticks and tick-borne pathogens. Our previous study has identified the actin and ferritin promoter regions in the Haemaphysalis longicornis tick.

Results: Here, the ferritin-derived promoter from the H. longicornis tick was characterized in silico, and the core promoter sequences and some of its important components were identified. Several truncations of the promoter region were created and inserted to a reporter plasmid to determine the important components for its activity. The activities of the truncated promoters on the Ixodes scapularis tick cell line (ISE6) were measured via a dual luciferase assay using experimental and control reporter genes. To induce the promoter's activity, transfected ISE6 cells were exposed to ferrous sulfate. The 639 nucleotides truncated promoter showed the highest activity on ISE6 cells when exposed to 1 mM ferrous sulfate.
\end{abstract}

Conclusion: In this study, we characterized an iron-inducible tick promoter that could be a valuable tool in the development of a gene-manipulation system to control ticks and tick-borne pathogens.

Keywords: Tick, Promoter, Ferritin, Dual-reporter plasmid, Transfection, Post-transcriptional regulation, ISE6 cells

\section{Background}

Next to mosquitoes, ticks are considered to be the most important vectors of pathogens that cause human diseases [1]. At present, the main method of controlling tick infestation has been the use of chemical acaricides. Due to numerous reports of acaricide resistance in ticks, alternative approaches are warranted [2]. Gene-manipulation techniques have been used in mosquitoes and have resulted in a decrease in mosquito populations and in the transmission of mosquito-borne pathogens [3]. However,

\footnotetext{
*Correspondence: k6199431@kadai.jp

1 Laboratory of Infectious Diseases, Joint Faculty of Veterinary Medicine,

Kagoshima University, 1-21-24 Korimoto, Kagoshima 890-0056, Japan

Full list of author information is available at the end of the article
}

gene-manipulation techniques in ticks mainly involved RNA interference (RNAi). RNAi techniques for specific tick molecules have been used mostly in the analysis of tick biological function and the interaction of ticks and tick-borne pathogens but not as the main method to control them [4]. This may be because RNAi is low throughput, labor-intensive, and relatively slow to yield results [5]. Therefore, an alternative gene-manipulation technique such as introduction of foreign genes in the ticks, could be explored to control ticks and tick-borne pathogens.

Haemaphysalis longicornis is a hard tick that can be found in East Asia and Australia and that has recently attracted much attention because of reports of infestation with this species in several states in the USA [6]. It is 
also considered a vector of the severe fever with thrombocytopenia syndrome virus (SFTSV) affecting humans [7, 8]. Numerous studies on $H$. longicornis, from its survival against acaricides to its vector competence against several viruses, have already been conducted $[9,10]$. It is necessary to control this tick species due to its effects on human and animal health and the economy. One possible method of tick and tick-borne disease control is the development of transgenic ticks.

The development of transgenic ticks requires an effective promoter derived from the organisms themselves. In mosquitoes, it is believed that the best candidate promoters for the expression of foreign genes are those that are inducible during blood-feeding due to their strength, tissue specificity, and synchrony of expression with the pathogen infection [11]. This could also be the case in ticks. Our previous study has identified two promoters from $H$. longicornis: the actin and ferritin1 promoters. The actin promoter could effectively express the foreign gene in an Ixodes scapularis embryo-derived cell line (ISE6), but the ability of the ferritin1 promoter to express a foreign gene was not demonstrated. It was hypothesized that this is due to the presence of iron-regulatory proteins (IRPs) in the untranslated region that prevents effective translation of the foreign gene [12]. On the other hand, our recent study showed a dose-dependent ferritin expression on a tick cell line after the addition of iron [13].

During blood-feeding, the actin gene and protein of ticks are constitutively expressed $[14,15]$. In the same manner, the ferritin1 mRNA is constitutively expressed, however, its protein is inducible by blood-feeding due to the presence of iron in the blood diet [14]. Therefore, the ferritin promoter could be a good candidate in the development of an inducible promoter system for the tick cell line that could be similar to the blood-feeding in ticks. Here, the promoter region of the ferritin1 gene of $H$. longicornis (HlFerl) was analyzed and its activity demonstrated using the ISE6 cell line.

\section{Methods}

In silico analysis of $\mathrm{H}$. longicornis ferritin 1 (HIFer 1 ) promoter sequence

Analysis of the HlFer 1 promoter region was conducted to determine the functional region of the previously identified HlFer1 promoter. Transcription starting site (TSS) analysis was done by first aligning the ESTs for HlFer 1 using MAFFT software (https://mafft.cbrc.jp/alignment/ software/). Then a WebLogo image of the TSS was made (https://weblogo.berkeley.edu/logo.cgi). Using the neural network promoter program (http://www.fruitfly.org/ seq_tools/promoter.html), the possible promoter regions were identified. Further characterization of the promoter regions was done using gene promoter-mining software (http://gpminer.mbc.nctu.edu.tw/).

\section{Renilla luciferase reporter construct}

To establish a purely tick promoter reporter plasmid, the H. longicornis actin (HlActin) promoter was used to replace the SV40 promoter of the Renilla luciferase (hrLuc) of the pmirGLO plasmid vector (Promega, Madison, WI, USA). First, the previously identified HlActin promoter region was amplified from the previously constructed pmirGLO-HlActin pro [12] using a primer pair of HlActin-Renilla $\mathrm{F}$ and HlActin-Renilla R (Additional file 1: Table S1) by PCR with KOD-Plus-Neo (Toyobo, Osaka, Japan). After amplification, the PCR products were electrophorosed in 1.0\% agarose gel. The PCR product was purified using a QIAquick Gel Extraction Kit (Qiagen, Hilden, Germany). The pmirGLO vector was then double-digested using $K p n \mathrm{I}$ and $P f m \mathrm{I}$ to remove the SV40 promoter. After double digestion, the plasmid vector was electrophoresed in 1.0\% agarose gel and purified using a QIAquick Gel Extraction Kit (Qiagen). The digested vector and purified product were mixed with an In-Fusion HD cloning kit (Takara, Shiga, Japan). After the ligation of pmirGLO/HlActin-hrLuc, the plasmid was transformed into the Escherichia coli Stellar strain and purified using a Plasmid Midi Kit (Qiagen).

\section{Firefly luciferase construct}

The previously constructed pmirGLO/HlActin-hrLuc was digested using BglII and HindIII at $37{ }^{\circ} \mathrm{C}$ for $2 \mathrm{~h}$ to remove the PGK promoter. After double digestion, the plasmid vector was electrophoresed in 1.0\% agarose gel and purified using a QIAquick Gel Extraction Kit (Qiagen). The HlFer 1 promoter sequence was amplified from the previously constructed pmirGLO-HlFer 1 plasmid using KOD-Plus-Neo (Toyobo) with the indicated primer sets (Additional file 1: Table S1). The HlFer1 promoter sequences were purified using a QIAquick Gel Extraction Kit (Qiagen). The purified vector was mixed with and ligated using an In-Fusion HD cloning kit (Takara). After ligation, the plasmids were transformed into the $E$. coli Stellar strain and purified using a Plasmid Midi Kit (Qiagen). For the pmirGLO-no promoter-Luc2/HlActinhrLuc, the purified vector was allowed to self-ligate using DNA Ligation Kit Ver 2.1 (Takara) and incubated at $16{ }^{\circ} \mathrm{C}$ overnight. It was then transformed into the $E$. coli DH5 $\alpha$ strain and purified using a Plasmid Midi Kit (Qiagen). Finally, the pmirGLO/HlFer1(F0-F4 and no promoter)Luc2/HlAct-hrLuc plasmids were obtained. Plasmid inserts from the clones were sequenced from the beginning to the end of the target promoter sequence using a BigDye Terminator v. 3.1 Cycle Sequencing Kit (Applied Biosystems, Foster City, CA, USA) with sequencing 
primers derived from the pmirGLO vector. A diagram for the constructed plasmid was created using SnapGene Viewer software (http://www.snapgene.com) (Fig. 1).

\section{Tick cell culture and transient transfection of plasmid vectors}

The ISE6 cell line from the embryo of I. scapularis was grown at $34{ }^{\circ} \mathrm{C}$ in $\mathrm{L}-15 \mathrm{~B}$ medium ( $\mathrm{pH}$ 6.4-6.6) with $10 \%$ fetal bovine serum, $5 \%$ tryptose phosphate broth, and 50 units/ml antibiotics-penicillin $\mathrm{G}$ and $50 \mu \mathrm{g} / \mathrm{ml}$ streptomycin $[16,17]$.

ISE6 cells were seeded in a 24 -well plate at $0.5 \mathrm{ml} /$ well of $1.0 \times 10^{6}$ cells $/ \mathrm{ml}$ and incubated at $34^{\circ} \mathrm{C}$ overnight. The plasmid vector $(1.5 \mu \mathrm{g} /$ well $), 50 \mu \mathrm{l}$ of Opti-MEM (Gibco, Grand Island, NY, USA), and $1.5 \mu \mathrm{l}$ of Lipofectamine 2000 (Invitrogen, Carlsbad, CA, USA) were mixed and incubated at room temperature for $5 \mathrm{~min}$. Then the incubated mixture was added to the medium in each well, and the cells were incubated at $34{ }^{\circ} \mathrm{C}$ for 2 days and replaced with different concentrations $(0,0.1,1$ and $2 \mathrm{mM}$ ) ferrous sulfate-enriched iron media, then further incubated for 4 days according to previous studies of ferritin induction [13]. The transfected cells were harvested and assayed for firefly and Renilla luciferase activities as described below.

\section{Dual luciferase assays}

The firefly and Renilla luciferase activities were assayed using a Dual-Glo Luciferase Assay System with Passive Lysis Buffer (Promega) and the microplate reader (SH9000Lab, Corona Electric, Ibaraki, Japan), following the manufacturer's protocol. The firefly luciferase activity corresponding to the Renilla luciferase activity was calculated (relative luciferase activity).

\section{Construction of yellow fluorescent protein (Venus) expression plasmid vector using HIFer 1 promoter regions}

Yellow fluorescent protein (Venus) expression vectors with an HlFer1(F2) promoter region were constructed. To produce a pmirGLO/Fer1 (F2)-Venus plasmid, the

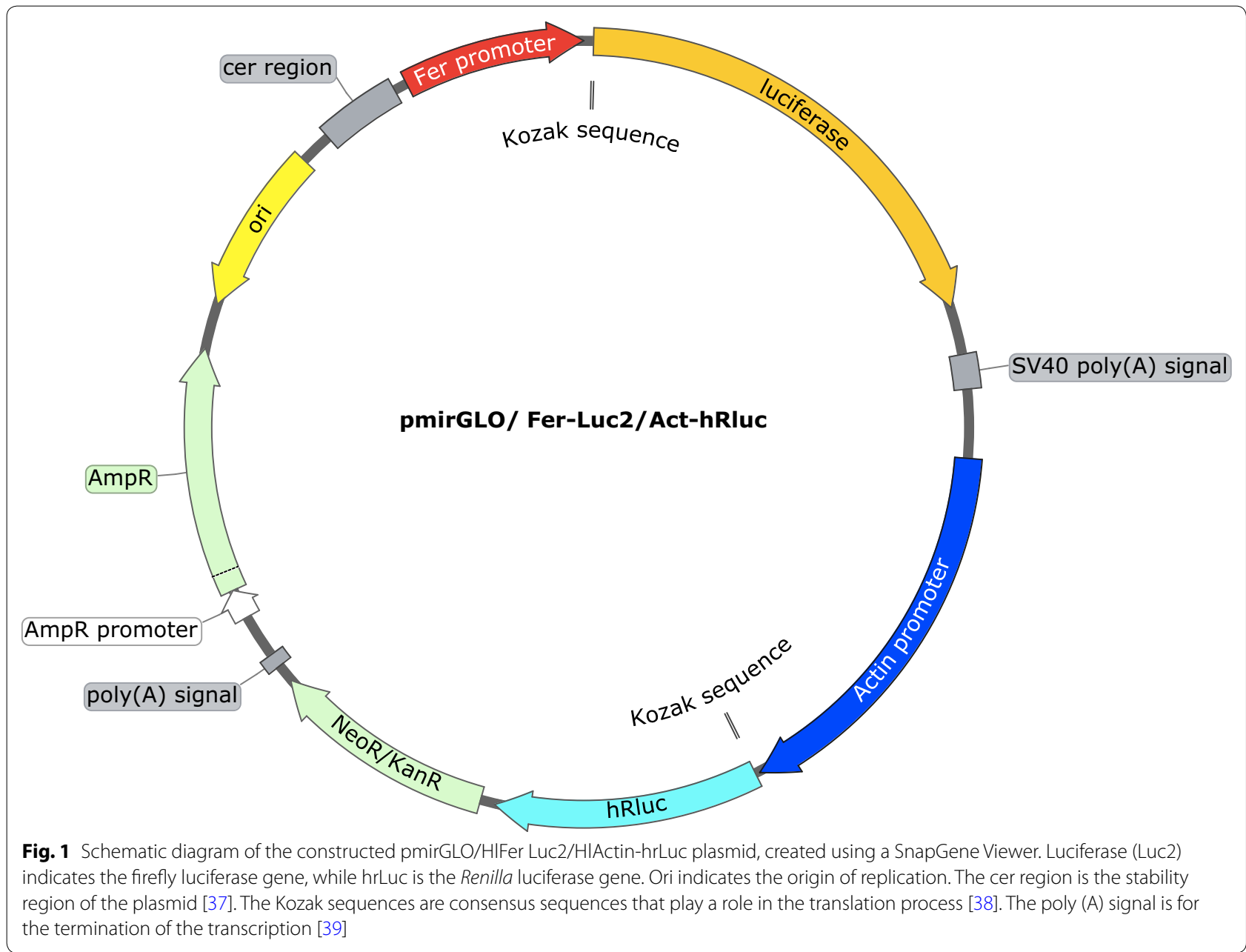


Fer1 (F2) promoter was amplified by PCR using KODPlus-Neo (Toyobo) with pmirGLO-HIFer2-Venus F and pmirGLO-HlFer2-Venus R primers, while the Venus gene was also amplified using pmirGLO-Venus $\mathrm{F}$ and pmirGLO Venus-XhoI R. These PCR products were purified using a QIAquick Gel Extraction Kit (Qiagen). The pmirGLO plasmid was double digested with $B g l \mathrm{II}$ and $\mathrm{XhoI}$ to remove the PGK promoter and luciferase gene from the vector. The obtained vector and two purified products were mixed with an In-Fusion HD Cloning Kit (Takara) and incubated. After ligation, the plasmid was transformed into the E. coli Stellar cells and purified using a Plasmid Midi Kit (Qiagen). The pmirGLO/no promoterVenus was constructed by blunt ligation of the purified Venus gene with the digested vector using DNA Ligation Kit Vector 2.1 (Takara).

\section{Transfection of Venus expression plasmid into ISE6 cells and comparison of promoter activities using fluorescence microscopy and western blotting}

Transfection was performed as mentioned above. As a negative control, Lipofectamine 2000 (Invitrogen) was used without the plasmid. The previously synthesized pmirGLO-HlActin-Venus was used as a positive control. The incubated mixture was added to each well, and after 2 days, the media were replaced with $1 \mathrm{mM}$ ferrous sulfate-enriched media and incubated at $34{ }^{\circ} \mathrm{C}$ for 4 days according to previous studies for promoter activity [5]. The transfected cells were observed under fluorescence microscopy (IX71, Olympus, Tokyo, Japan).

Another set of transfected cells was collected and subjected to western blotting as described by Kusakisako et al. [12]. Briefly, transfected cells were collected and suspended in phosphate-buffered saline (PBS) and sonicated at $45 \mathrm{kHz}$ for 6 min using a VS-100III ultrasonic cleaner (AS ONE, Osaka, Japan), then centrifuged at 20,100 $\times g$. The supernatant was resolved in a $12 \%$ SDS-polyacrylamide gel electrophoresis (SDS-PAGE) under a reducing condition. The proteins were then transferred onto a polyvinylidene difluoride membrane (Immobilon-P; Millipore, Danvers, MA, USA). The membranes were then blocked at room temperature for $1 \mathrm{~h}$ with $0.3 \%$ skimmed milk in PBS containing 0.05\% Tween 20 (PBS-T, blocking solution). After blocking, the membranes were incubated in a 1:1000 dilution anti-green fluorescent protein (GFP) pAb (MBL, Nagano, Japan) in a blocking solution at $4{ }^{\circ} \mathrm{C}$ overnight. The antiserum against recombinant $H$. longicornis $\beta$-tubulin [18] was used as a loading control. After incubation, the membranes were washed three times with PBS-T and then incubated with a 1:50,000 dilution of horseradish peroxidase (HRP)-conjugated goat antimouse or anti-rabbit IgG (Dako, Glostrup, Denmark) in a blocking solution at room temperature for $1 \mathrm{~h}$. The membranes were then washed five times using PBS-T. After washing, the bands were detected using Amersham $^{\mathrm{TM}} \mathrm{ECL}^{\mathrm{TM}}$ Prime Western Blotting Detection Reagent (GE Healthcare, Buckinghamshire, UK) and viewed using FluorChem ${ }^{\circledR} \mathrm{FC} 2$ software (Alpha Innotech, San Leandro, CA, USA).

\section{Statistical analysis}

Statistical analyses were performed using STATA15.0 software. The data were initially checked for normality and homogeneity assumptions using the Shapiro-Wilk W-test for normality and Breusch-Pagan/Cook-Weisberg test for heteroskedasticity. A one-way analysis of variance (ANOVA) with Bonferroni multiple comparison posthoc tests was applied. Statistical significance was set as $P<0.05$. Data presented are the results of at least two independent experiments.

\section{Results \\ Analysis of the Haemaphysalis longicornis ferritin 1 promoter region sequence}

To determine the core promoter region of the previously identified HlFer1 promoter sequence [12], analysis of the promoter sequence was performed. Initially, the transcription starting site (TSS) was identified. Alignment of HlFer 1 candidate sequences from the expressed sequence tag (EST) library was performed using MAFFT alignment software (Fig. 2a). Based on the alignment, a WebLogo image of the TSS region was made (Fig. 2b). To further investigate the core promoter sequence containing the TSS region of the HlFerl gene, the sequence of the HlFer 1 promoter region was subjected to a neural network for promoter prediction (NNPP) program in the Berkeley Drosophila Genome Project. Based on the alignment and the prediction software, the $[\mathrm{T}]$ nucleotide was the estimated TSS among the sequences (Figs. 2 and 3a).

The NNPP program also predicted several promoter sequences from the HlFer 1 promoter region (Fig. 3a). This promoter region was also evaluated using promotermining software to determine the important components of the promoter regions, such as the TATA and GC box, and match them with the predicted promoter sequence. Based on the findings, five promoter sequences were identified as candidates for an optimal promoter region of HlFer 1 and tentatively labeled as HlFer 1 (F0) for the 2848 nucleotide (nt) whole promoter, HlFer1 (F1) for the 821 nt promoter, HlFer1 (F2) for the 638 nt promoter, HlFer1 (F3) for the $450 \mathrm{nt}$ promoter, and HlFer1 (F4) for the $185 \mathrm{nt}$ promoter (Fig. $3 \mathrm{~b}$ ). These results indicate that the identified promoter region has a high possibility of being the true promoter region of HlFerl. 


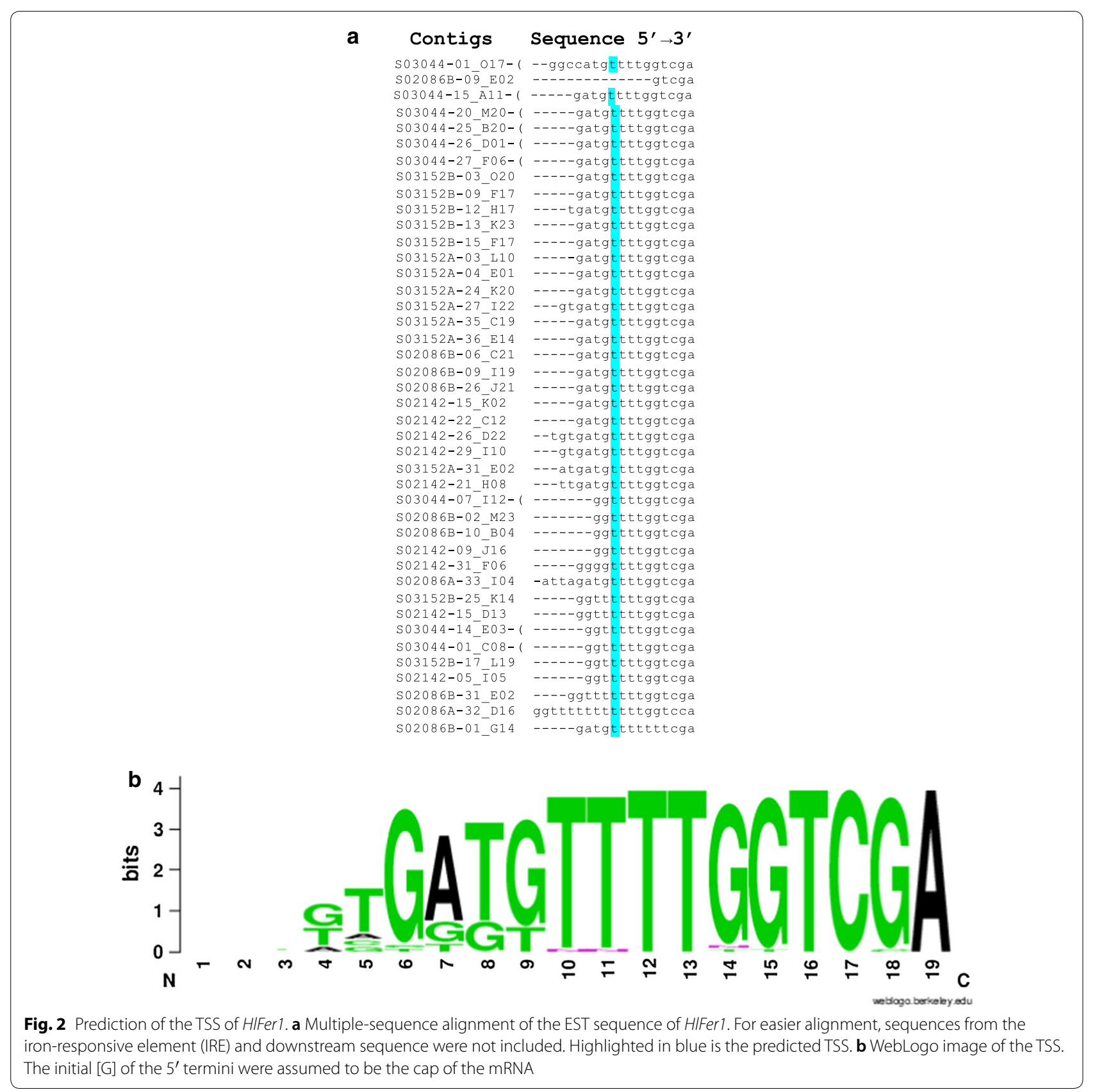

\section{Evaluation of the HIFer 1 promoter sequences activity in ISE6 cells using a dual luciferase assay}

Promoter-replacing expression vectors were constructed based on a pmirGLO plasmid vector with the phosphoglycerate kinase (PGK) promoter as the original promoter. The PGK promoter was replaced by truncated HlFer 1 promoters to evaluate the promoter activity of the candidate HlFer 1 promoter sequences in ISE6 cells. The previously identified HlActin promoter was used instead of the SV40 promoter to maintain consistency in using a tick-derived promoter. It has been previously demonstrated that the interaction between the ironresponsive element (IRE) and IRP may have resulted in the depressed activity of the HlFer 1 promoter. Therefore, different concentrations of ferrous sulfate were used in the media to liberate the IRP from the IRE for translation induction. A dose-dependent luciferase activity for the different truncated promoters up to $1 \mathrm{mM}$ ferrous sulfate was observed. With $2 \mathrm{mM}$ ferrous sulfate, decreased activity was observed at the HlFer1 (F1), HlFer1 (F2), 


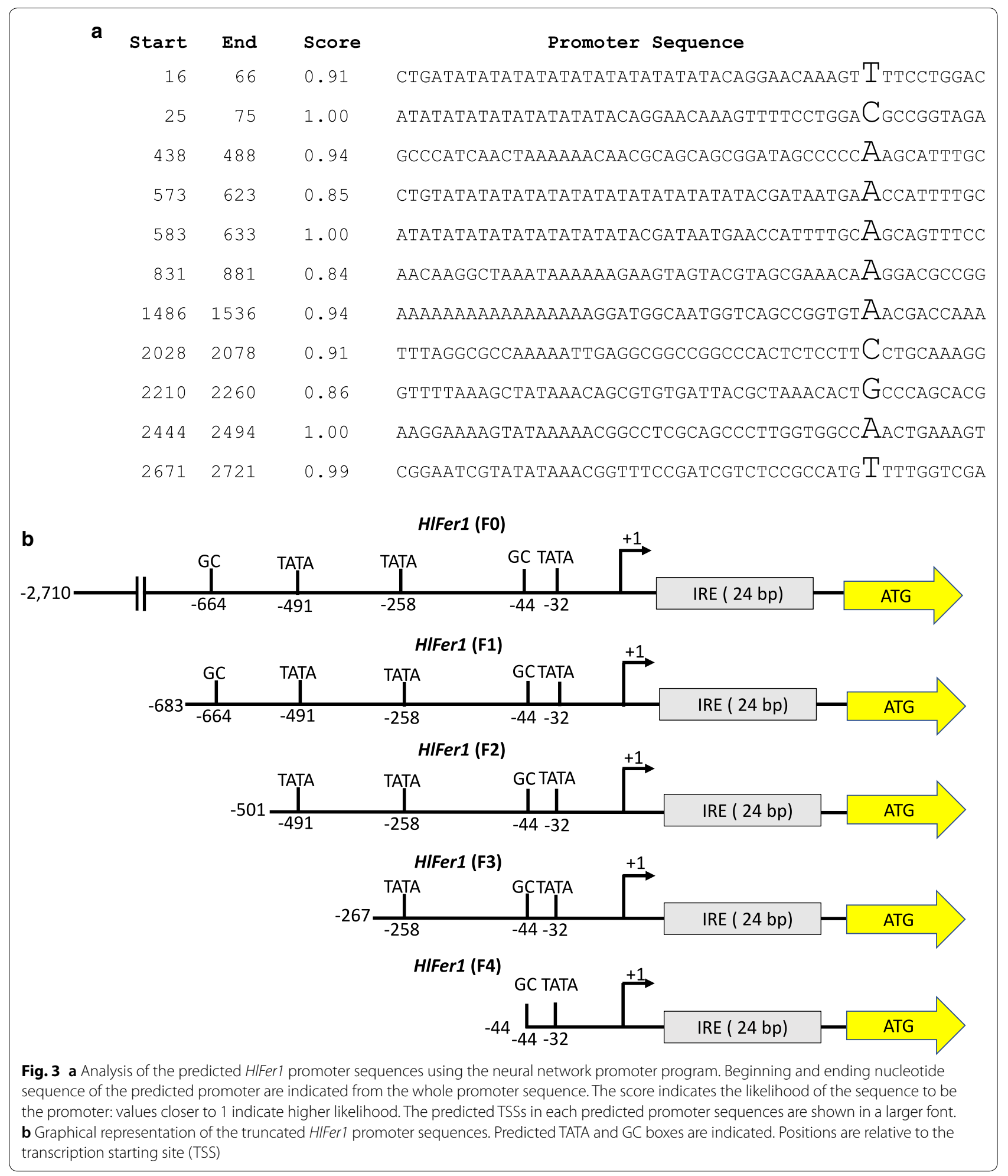

and HlFer1 (F3) truncates (Fig. 4, Additional file 1: Table S2). Among the promoters, the HlFer1 (F2) possessed the highest luciferase activity in all of the ferrous iron-enriched media, followed by the HlFer1 (F1) and
HlFer1 (F3) truncates, except at a $2 \mathrm{mM}$ ferrous sulfate concentration, wherein HlFer1 (F3) has a slightly higher activity than HlFer1 (F1). The HlFer 1 (F0) and HlFer1 (F4) sequences exhibited the lowest relative luciferase activity, 


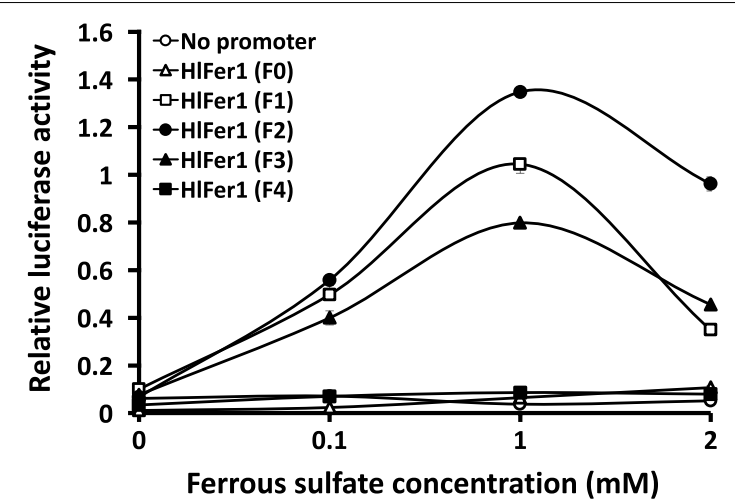

Fig. 4 Evaluation of the HIFer 1 promoter truncates using relative luciferase activity in ISE6 cells enriched with different concentrations of ferrous sulfate. The pmirGLO plasmids containing HIFer 1 promoter truncates were transfected to ISE6 cells using a 1:1 Lipofectamine 2000 transfection reagent. The cells were incubated for 2 days, replaced with different concentrations of ferrous sulfate $(0,0.1,1$ and $2 \mathrm{mM}$ ) enriched media, incubated for another 4 days, and then tested for luciferase activity using a Dual-Glo Luciferase Assay System following the manufacturer's protocol

and their relative luciferase activity did not differ significantly (Fig. 4, Additional file 1: Table S2). These results demonstrate that the HlFer 1 (F4) being the smallest truncated promoter and still exhibited activity could be the core promoter region, while the HlFer1 (F2) showed optimal promoter activity in the presence of $1 \mathrm{mM}$ ferrous sulfate $\left(F_{(15,24)}=540.83, P<0.0001\right)$.

\section{Demonstration of the promoter activity of the HIFer1 promoter regions in ISE6 cells under fluorescence microscopy and western blotting}

Expression vectors of a yellow fluorescent protein, Venus, were constructed to evaluate the HlFerl (F2) promoter activity under fluorescence microscopy to show the activity of this truncated promoter. The previously constructed pmirGLO-HlAct pro-Venus and pmirGLO-HlFer1(F2)Venus, wherein the PGK promoter and luciferase gene were replaced by the HlFer1(F2) promoter and the Venus gene, respectively, in the pmirGLO plasmid were evaluated. The mentioned vectors were transfected to ISE6 cells, and then the cells were enriched with $1 \mathrm{mM}$ ferrous sulfate. The fluorescence of Venus was observed under fluorescence microscopy at day 4 after ferrous sulfate enrichment. The fluorescence microscopy test showed that the addition of $1 \mathrm{mM}$ ferrous sulfate resulted in the increased fluorescence intensity of pmirGLO-HlFer 1(F2)Venus transfected cells; however, the addition of $1 \mathrm{mM}$ ferrous sulfate did not affect the intensity of pmirGLOHlAct pro-Venus transfected cells. No apparent difference in intensity was observed between pmirGLO-HlAct pro-Venus and pmirGLO-HlFer1(F2)-Venus transfected
ISE6 cells (Fig. 5a). Venus proteins were also investigated using western blotting. Western blot analysis showed that the addition of $1 \mathrm{mM}$ ferrous sulfate to the ISE6 cells transfected with pmirGLO-HlFer1(F2)-Venus resulted in expression of the Venus protein in ISE6 cells (Fig. 5b). These results demonstrate that the promoter activity for a foreign gene is not limited to the luciferase gene but also occurs in other genes, such as the Venus gene.

\section{Discussion}

Genetic manipulation of mosquitoes, particularly the introduction of foreign gene, has been shown to impair the transmission of malaria parasites [11, 19]. The development of ticks that possess genes that could affect the infection and transmission of tick-borne pathogens, then, could be explored as a promising strategy to control tick-borne diseases. Since gene regulation is a critical, well-coordinated, and complex process, an effective promoter is essential for the expression of a foreign gene into the target organism [12]. An inducible promoter is also important in the development of a tunable recombinant expression system [20, 21]. Recombinant proteins are important in the development of tick control strategies especially in vaccine design [22]. Insect cell lines are frequently utilized in recombinant protein production [23]. This strategy could be explored in tick cell lines in the development of expression system for tick-specific recombinant proteins.

Promoters are considered to be gene-regulatory sequences found immediately upstream of the TSS [24]. In this study, the promoter region of HlFerl was characterized for its possible use in the development of transgenic ticks and recombinant protein expression system in tick cell lines.

The core promoter is the minimal DNA sequence that is sufficient to initiate gene transcription [25]. It is where the transcription machinery is assembled [26]. The core promoter is generally 50 to $100 \mathrm{nt}$ within the TSS [24]. Using the EST database promoter analysis software, the TSS [T] is predicted to be 138-bp upstream of the open reading frame sequence in the HlFerl gene. The TSS is included in the initiator element, which is, in turn, a key feature of the core promoter sequence [25]. Aside from the initiator element, another common core promoter element is the TATA box [26]. In metazoans, the TATA box is usually located around $30 \mathrm{nt}$ upstream of the TSS, wherein the optimal spacing between the +1 TSS and the first $\mathrm{T}$ is around $30-31 \mathrm{nt}$ upstream $[25,26]$. The TATA sequence serves as the binding site for the transcription factor II D (TFIID). In this study, the TATA box was predicted to be $32 \mathrm{nt}$ upstream of the TSS (Fig. 3b). Based on these results, the F4 sequence promoter was 


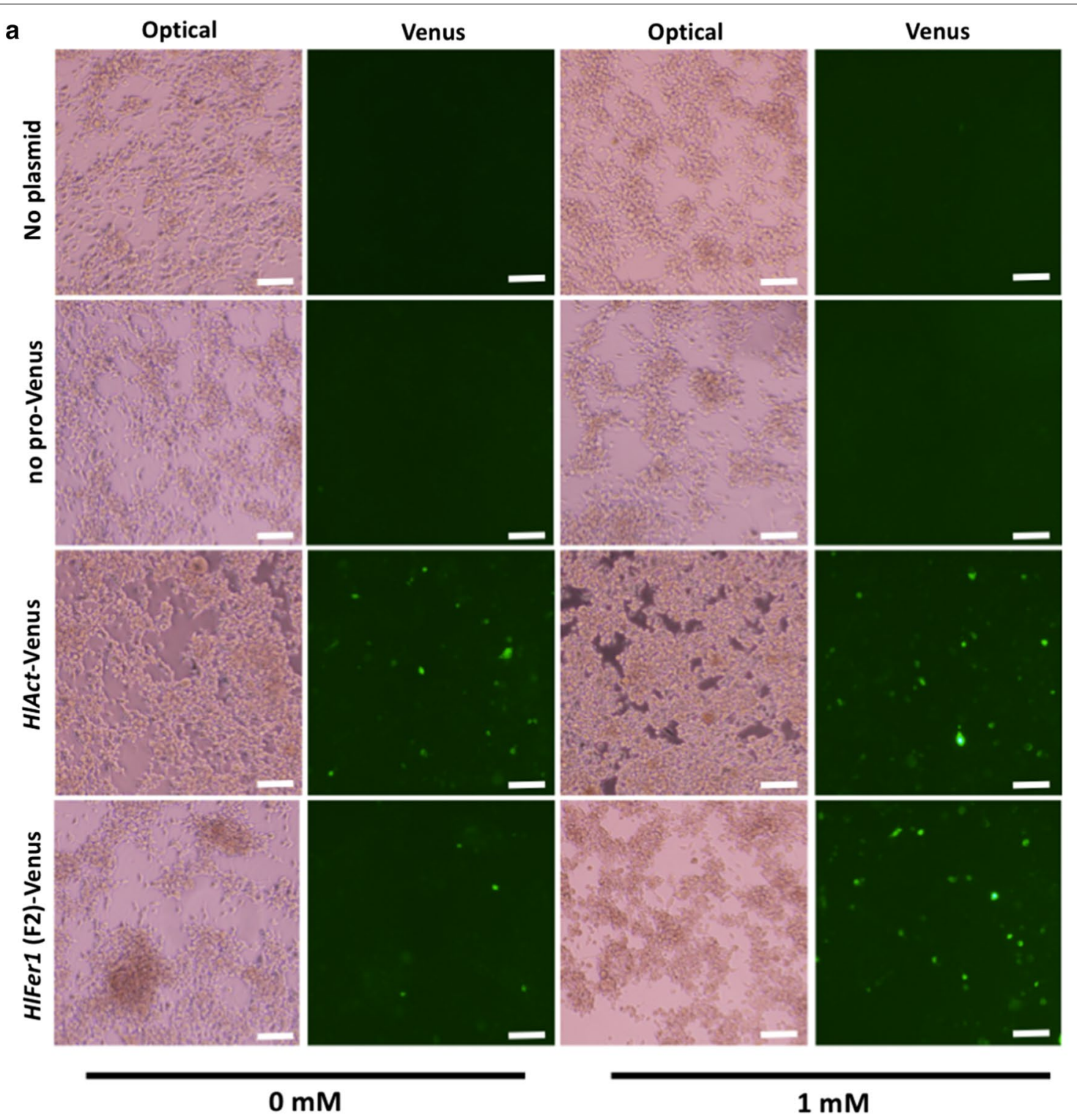

b

Ferrous sulfate concentration ( $\mathrm{mM}$ )

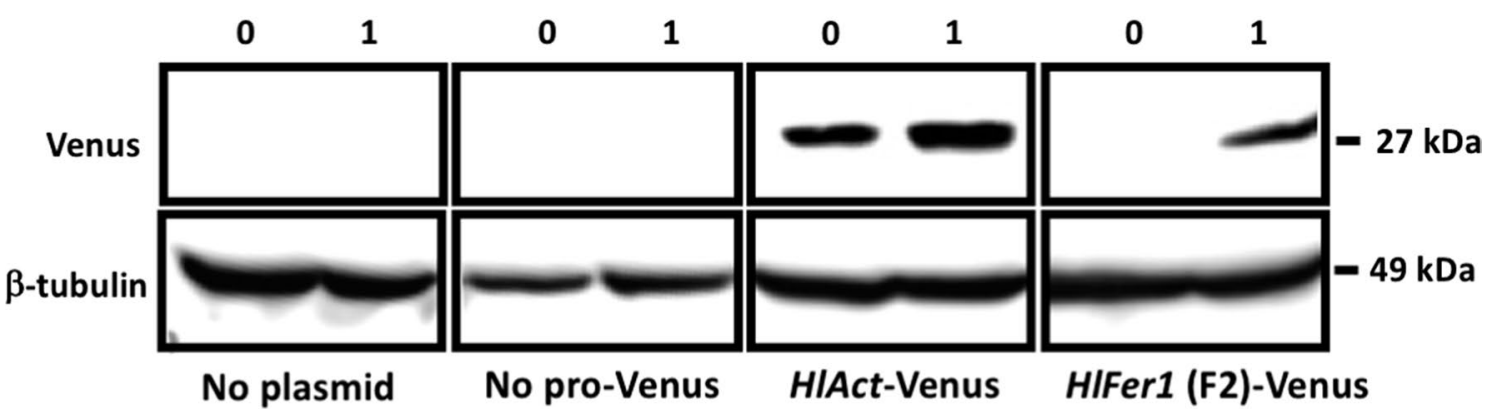

Fig. 5 Observation of HIFer1 (F2) promoter activity using Venus in ISE6 cells via fluorescence microscopy (a) and western blotting (b). The pmirGLO-no promoter-Venus, pmirGLO-HIAct pro-Venus, and pmirGLO-HIFer1(F2)-Venus plasmids (1.5 $\mu \mathrm{g}$ ) with $1.5 \mu$ I Lipofectamine 2000 were transfected into $1 \times 10^{6} \mathrm{ISE} 6$ cells in a 24-well plate. Media enriched with $1 \mathrm{mM}$ ferrous sulfate were used 2 days after transfection. Cells were observed under a fluorescence microscope after 4 days of medium enrichment. Western blotting results are representative data of three separate experiments. Scale-bars: $100 \mu \mathrm{m}$ 
hypothesized as part of the core promoter region of the HlFer1 promoter.

In vitro studies have shown that the core promoter is sufficient to initiate promoter activity, but an upstream promoter sequence may be necessary to generate activity in vivo [27]. The upstream sequence is also known as the extended promoter sequence. The extended promoter sequence is considered to contain regulatory elements that can modulate the downstream gene sequences [24, 26]. Therefore, several HlFer 1 sequences upstream of the core promoter region were evaluated using a reporter assay to determine the promoter sequence with the optimal activity (Fig. 3b).

A dual reporter assay, such as the dual luciferase reporter assay, has been commonly used in cell lines to evaluate promoter activity [12, 28, 29]. The use of a dual reporter system has been very advantageous in measuring gene expression. It is sensitive, allows experimental variations due to the presence of a control reporter gene, and has a high-throughput platform [30]. The dual luciferase assay has also been shown to be of value in tick cell lines [5] and has been used in evaluation of tick promoter. In this experiment, we used the previously identified HlAct promoter sequence from the H. longicornis tick [12] as the promoter of the control reporter gene and the HlFer 1 promoter sequences for the experimental reporter gene. Since there are no available cell lines for the $H$. longicornis tick, the embryo-derived $I$. scapularis cell line (ISE6) was utilized to evaluate the core promoter sequence of the different candidates in the HIFer1 promoter sequences since the activity of HlAct and HlFer1 promoter has already been established in this tick cell line [12].

Although the HlFer 1 promoter was tested for activity in our previous study, it showed depressed or no transcriptional activity [12]. This could be attributed not to the absence of transcriptional activity but rather to the presence of an IRE in the HlFer1 mRNA, which interacts with the IRP that hinders the protein translation. On a previous study, an increasing HIFer1 protein expression was observed during blood-feeding despite no difference in the expression of HlFer 1 mRNA expression. It was then hypothesized that the increasing expression was due to the liberation of IRP from IRE due to the increased iron in the tick blood meal [14]. The same mechanism of intracellular ferritin protein expression was also proposed in Ixodes ticks [31]. In our recent study on the ISE6 cell line, Fer1 protein expression was induced by the addition of 0.1 to 2 $\mathrm{mM}$ ferrous sulfate to the media and incubation for at least 2 days [13]. It could be safe to assume therefore that the mechanism of intracellular ferritin translation in Haemaphysalis and Ixodes ticks, as well as Ixodes cells are all regulated by iron concentration. Therefore, ferrous sulfate enrichment on HlFer 1 promoter transfected ISE6 cells was utilized to determine whether it would result in luciferase activity due to liberation of the IRP and eventually, protein translation. A dosedependent increase in activity was observed to peak in $1 \mathrm{mM}$ ferrous sulfate-enriched media. The lower luciferase activity with $2 \mathrm{mM}$ ferrous sulfate could be attributed to the possible increased cell mortality at the increased ferrous concentration, as was observed in our previous study [13]. With $1 \mathrm{mM}$ ferrous sulfate, the HlFer1 (F2) promoter showed the highest activity, followed by HlFer1 (F1), HlFer1 (F3), HlFer1 (F4), and HlFer1 (F0), with HlFer1 (F4) and HlFer1 (F0) not differing significantly, thus strengthening our hypothesis that the HlFer1 (F4) promoter is the core promoter region. Since we consider this region to be the core promoter region, its activity could also be considered the basal activity of the promoter region [24]. The increased promoter activity of the HlFer1 (F3) and HlFer1 (F2) promoter regions could indicate that these extended regions contain the enhancers or positive regulatory elements. The decrease in the activity of the HlFer1 (F1) and especially the HlFer1 (F0) region could indicate that these regions contain the silencers or negative regulatory elements. This is consistent with human cell lines, in which the -350 to $-40 \mathrm{nt}$ region showed enhanced transcriptional activity, while the region further upstream, especially -500 to $-1000 \mathrm{nt}$, showed a decline in transcriptional activity [24]. This is also consistent with tick promoters, wherein the promoter region $-649 \mathrm{nt}$ from the TSS of the ribosomal protein L4 (rpL4) promoter of Rhipicephalus microplus showed a 17 -fold higher promoter activity when compared with the 1191 nt originally cloned promoter. Meanwhile, a three-fold increase was observed when the -1005 to -495 sequences were deleted from the EF1- $\alpha$ promoter of the same tick species, indicating the presence of repressive elements in the upstream sequences of the promoters [5].

To further confirm the activity of the geneexpression vectors in ISE6 cells, Venus geneexpression vectors (pmirGLO/HlAct-Venus and pmirGLO/HlFer1(F2)-Venus) were constructed. More intense Venus fluorescence within transfected cells of the ferrous sulfate-enriched ISE6 cells was detected in pmirGLO-HlFer1 (F2)-Venus transfected groups as compared to those without ferrous sulfate enrichment. Meanwhile, no difference in the ferrous sulfate-enrichment and nonenrichment was observed on pmirGLO-HlAct pro-Venus transfected cells. It would then be of interest to know if this Fer1 promoter sequence could also be found in $I$. scapularis and would exhibit same or higher activity. 
Nonetheless, we believed that the HlFer1 promoter sequence maintained its promoter activity. These results also demonstrate the iron inducibility of the activity of the ferritin derived promoter.

\section{Conclusions}

Precise spatial and temporal gene-expression and genemodification control is necessary in the study of several gene functions. An inducible system of gene expression has been a valuable tool in the conduct of such study [32]. The use of iron as an inducing agent for promoter activity has already been demonstrated in Trichomonas vaginalis $[33,34]$. Ticks, being obligate hematophagous parasites, are exposed to large amounts of iron and have developed mechanisms to overcome iron-related toxicity $[35,36]$. We took advantage of presence of an IRE in the intracellular ferritin mRNA to identify and develop an ironinducible promoter that could be used in tick cell line. Since many tick-borne pathogens are acquired during blood-feeding, a blood-feeding inducible tick promoter is necessary to the development of transgenic ticks for tick-borne pathogen control. Since blood-feeding would be difficult to simulate in tick cells, an increased iron environment would be a good alternative, since there is also an increased iron intake during blood-feeding. In this case, an iron-inducible promoter could be a valuable tool in such studies. The promoter could then be used in cell lines for the study of antimicrobial peptides against tick-borne pathogens that could be expressed during blood-feeding in the development of transgenic ticks. Aside from the abovementioned, this promoter could be applied for the development of an expression system that could be used to produce recombinant proteins that are tick-specific.

\section{Additional file}

Additional file 1: Table S1. Oligonucletiode primer sequences used for construction of the plasmids. Table S2. Relative luciferase activity of the different HIFer1 promoter truncates in ISE6 cells exposed to different concentrations of ferrous sulfate.

\section{Abbreviations \\ ISE6: IXodes scapularis embryo-derived tick cell line; RNAi: RNA interference; HIFer1: Haemaphysalis longicornis ferritin 1; TSS: transcription starting site; EST: expressed sequence tags; NNPP: neural network for promoter prediction; PGK: phosphoglycerate kinase; IRE: iron-responsive element; IRP: iron-regulatory protein; TFIID: transcription factor II D; PBS: phosphate-buffered saline; HRP horseradish peroxidase; ANOVA: analysis of variance; Luc2: firefly luciferase; GFP: green fluorescent protein.}

\section{Acknowledgements}

We would like to acknowledge Dr Ulrike Munderloh for kindly providing us with the ISE6 cells. We also would like to acknowledge the assistance of Dr Rodel Jonathan S. Vitor II of the Laboratory of Veterinary Pathophysiology and
Animal Health, Graduate School of Agricultural and Life Sciences, The University of Tokyo, especially in analyzing the data.

\section{Authors' contributions}

$\mathrm{EPH}, \mathrm{TH}$, and TT designed the experiments. EPH and KK performed the experiments. EPH, TH, and TT analyzed the data. EPH wrote the manuscript. All authors read and approved the final manuscript.

\section{Funding}

This study was supported by the Japan Society for the Promotion of Science (JSPS) KAKENHI Grant Numbers 15H05264, 16H05028, and $17 \mathrm{~K} 19328$ and the Takeda Science Foundation. E. P. Hernandez was supported by the Japanese Government's Ministry of Education, Culture, Sports, Science and Technology Scholarship (Monbukagakusho: MEXT) for doctoral fellowship. The funding bodies had no role in the design of the study; in the collection, analysis, and interpretation of data; or in writing the manuscript.

\section{Availability of data and materials}

All data generated or analyzed during this study are included in this published article and its additional file.

\section{Ethics approval and consent to participate}

Not applicable.

\section{Consent for publication}

Not applicable.

\section{Competing interests}

The authors declare that they have no competing interests.

\begin{abstract}
Author details
${ }^{1}$ Laboratory of Infectious Diseases, Joint Faculty of Veterinary Medicine, Kagoshima University, 1-21-24 Korimoto, Kagoshima 890-0056, Japan.

${ }^{2}$ Department of Pathological and Preventive Veterinary Science, The United Graduate School of Veterinary Science, Yamaguchi University, Yoshida, Yamaguchi 753-8515, Japan. ${ }^{3}$ Laboratory of Parasitology, Graduate School of Veterinary Medicine, Hokkaido University, Sapporo, Hokkaido 060-0818, Japan. ${ }^{4}$ Department of Parasitology, Kitasato University School of Medicine, Kitasato, Minami, Sagamihara, Kanagawa 252-0374, Japan.
\end{abstract}

Received: 6 May 2019 Accepted: 19 June 2019

Published online: 25 June 2019

\section{References}

1. de la Fuente J, Estrada-Pena A, Venzal JM, Kocan KM, Sonenshine DE. Overview: ticks as vectors of pathogens that cause disease in humans and animals. Front Biosci. 2008;13:6938-46.

2. Jongejan F, Uilenberg G. Ticks and control methods. Rev Sci Tech. 1994;13:1201-26.

3. de Paes Andrade P, Aragão FJL, Colli W, Dellagostin OA, Finardi-Filho F, Hirata $\mathrm{MH}$, et al. Use of transgenic Aedes aegypti in Brazil: risk perception and assessment. Bull World Health Organ. 2016;94:766-71.

4. de la Fuente J, Kocan KM, Almazán C, Blouin EF. RNA interference for the study and genetic manipulation of ticks. Trends Parasitol. 2007;23:427-33.

5. Tuckow AP, Temeyer KB. Discovery, adaptation and transcriptional activity of two tick promoters: construction of a dual luciferase reporter system for optimization of RNA interference in Rhipicephalus (Boophilus) microplus cell lines. Insect Mol Biol. 2015;24:454-66.

6. Beard CB, Occi J, Bonilla DL, Egizi AM, Fonseca DM, Mertins JW, et al. Multistate infestation with the exotic disease-vector tick Haemaphysalis longicornis_-United States, August 2017-September 2018. MMWR Morb Mortal Wkly Rep. 2018;67:1310-3.

7. Luo L-M, Zhao L, Wen H-L, Zhang Z-T, Liu J-W, Fang L-Z, et al. Haemaphysalis longicornis ticks as reservoir and vector of severe fever with thrombocytopenia syndrome virus in China. Emerg Infect Dis. 2015;21:1770-6.

8. Nuttall PA. Tick-borne viruses. In: Sonenshine DE, Roe RM, editors. Biology of Ticks. 2nd ed. New York: Oxford University Press; 2014. p. 180-210. 
9. Hernandez EP, Kusakisako K, Talactac MR, Galay RL, Hatta T, Fujisaki K, et al. Glutathione S-transferases play a role in the detoxification of flumethrin and chlorpyrifos in Haemaphysalis longicornis. Parasit Vectors. 2018;11:460.

10. Talactac MR, Yoshii K, Hernandez EP, Kusakisako K, Galay RL, Fujisaki K, et al. Vector competence of Haemaphysalis longicornis ticks for a Japanese isolate of the Thogoto virus. Sci Rep. 2018;8:9300.

11. Moreira LA, Ito J, Ghosh A, Devenport M, Zieler H, Abraham EG, et al. Bee venom phospholipase inhibits malaria parasite development in transgenic mosquitoes. J Biol Chem. 2002:277:40839-43.

12. Kusakisako K, Ido A, Masatani T, Morokuma H, Hernandez EP, Talactac MR, et al. Transcriptional activities of two newly identified Haemaphysalis longicornis tick-derived promoter regions in the Ixodes scapularis tick cell line (ISE6). Insect Mol Biol. 2018;27:590-602.

13. Hernandez EP, Kusakisako K, Talactac MR, Galay RL, Yoshii K, Tanaka T. Induction of intracellular ferritin expression in embryo-derived Ixodes scapularis cell line (ISE6). Sci Rep. 2018;8:16566.

14. Galay RL, Aung KM, Umemiya-Shirafuji R, Maeda H, Matsuo T, Kawaguchi $\mathrm{H}$, et al. Multiple ferritins are vital to successful blood feeding and reproduction of the hard tick Haemaphysalis longicornis. J Exp Biol. 2013:216:1905-15.

15. da Silva Vaz I, Imamura S, Nakajima C, de Cardoso FC, Ferreira CAS, Renard $\mathrm{G}$, et al. Molecular cloning and sequence analysis of cDNAs encoding for Boophilus microplus, Haemaphysalis longicornis and Rhipicephalus appendiculatus actins. Vet Parasitol. 2005:127:147-55.

16. Munderloh UG, Liu Y, Wang M, Chen C, Kurtti TJ. Establishment, maintenance and description of cell lines from the tick Ixodes scapularis. J Parasitol. 1994:80:533-43.

17. Yoshii K, Goto A, Kawakami K, Kariwa H, Takashima I. Construction and application of chimeric virus-like particles of tick-borne encephalitis virus and mosquito-borne Japanese encephalitis virus. J Gen Virol. 2008:89:200-11

18. Umemiya-Shirafuji R, Tanaka T, Boldbaatar D, Tanaka T, Fujisaki K. Akt is an essential player in regulating cell/organ growth at the adult stage in the hard tick Haemaphysalis longicornis. Insect Biochem Mol Biol. 2012:42:164-73.

19. Ito J, Ghosh A, Moreira LA, Wimmer EA, Jacobs-Lorena M. Transgenic anopheline mosquitoes impaired in transmission of a malaria parasite. Nature. 2002:417:452-5.

20. Lee S, Lee YJ, Choi S, Park S-B, Tran Q-G, Heo J, et al. Development of an alcohol-inducible gene expression system for recombinant protein expression in Chlamydomonas reinhardtii. J Appl Phycol. 2018;20:2297-304.

21. Fitz E, Wanka F, Seiboth B. The promoter toolbox for recombinant gene expression in Trichoderma reesei. Front Bioeng Biotechnol. 2018;6:135.

22. Galay RL, Miyata T, Umemiya-Shirafuji R, Mochizuki M, Fujisaki K, Tanaka T. Host immunization with recombinant proteins to screen antigens for tick control. Methods Mol Biol. 2016;1404:261-73.

23. Yu X, Hua J, Zhang J, Ma L. Expression of recombinant proteins in insect cells by their direct infection with Escherichia coli transformed with baculovirus bacmids. Acta Virol. 2014;58:61-8.
24. Cooper SJ, Trinklein ND, Anton ED, Nguyen L, Myers RM. Comprehensive analysis of transcriptional promoter structure and function in $1 \%$ of the human genome. Genome Res. 2006;16:1-10.

25. Butler JEF, Kadonaga JT. The RNA polymerase II core promoter: a key component in the regulation of gene expression. Genes Dev. 2002;16:2583-92.

26. Roy AL, Singer DS. Core promoters in transcription: old problem, new insights. Trends Biochem Sci. 2015;40:165-71.

27. Zhang MQ. Identification of human gene core promoters in silico. Genome Res. 1998;8:319-26.

28. Solberg N, Krauss S. Luciferase assay to study the activity of a cloned promoter DNA fragment. Methods Mol Biol. 2013;977:65-78.

29. Alcaraz-Pérez F, Mulero V, Cayuela ML. Application of the dual-luciferase reporter assay to the analysis of promoter activity in Zebrafish embryos. BMC Biotechnol. 2008;8:81.

30. McNabb DS, Reed R, Marciniak RA. Dual luciferase assay system for rapid assessment of gene expression in Saccharomyces cerevisiae. Eukaryot Cell. 2005:4:1539-49.

31. Hajdušek O, Sojka D, Kopáček P, Buresová V, Franta Z, Sauman I, et al. Knockdown of proteins involved in iron metabolism limits tick reproduction and development. Proc Natl Acad Sci USA. 2009;106:1033-8.

32. Jaisser F. Inducible gene expression and gene modification in transgenic mice. J Am Soc Nephrol. 2000;11(Suppl. 16):95-100.

33. Hsu H-M, Ong S-J, Lee M-C, Tai J-H. Transcriptional regulation of an ironinducible gene by differential and alternate promoter entries of multiple Myb proteins in the protozoan parasite Trichomonas vaginalis. Eukaryotic Cell. 2009:8:362-72.

34. Tsai C-D, Liu H-W, Tai J-H. Characterization of an iron-responsive promoter in the protozoan pathogen Trichomonas vaginalis. J Biol Chem. 2002;277:5153-62.

35. Galay RL, Umemiya-Shirafuji R, Mochizuki M, Fujisaki K, Tanaka T. Iron metabolism in hard ticks (Acari: Ixodidae): the antidote to their toxic diet. Parasitol Int. 2015;64:182-9.

36. Hajdušek O, Šíma R, Perner J, Loosová G, Harcubová A, Kopáček P. Tick iron and heme metabolism - new target for an anti-tick intervention. Ticks Tick Borne Dis. 2016;7:565-72.

37. Colloms SD, Sykora P, Szatmari G, Sherratt DJ. Recombination at ColE1 cer requires the Escherichia coli xerC gene product, a member of the lambda integrase family of site-specific recombinases. J Bacteriol. 1990;172:6973-80.

38. De Angioletti M, Lacerra G, Sabato V, Carestia C. Beta+45 G-> C: a novel silent beta-thalassaemia mutation, the first in the Kozak sequence. $\mathrm{Br}$ J Haematol. 2004;124:224-31.

39. Proudfoot NJ, Furger A, Dye MJ. Integrating mRNA processing with transcription. Cell. 2002;108:501-12.

\section{Publisher's Note}

Springer Nature remains neutral with regard to jurisdictional claims in published maps and institutional affiliations.

\footnotetext{
Ready to submit your research? Choose BMC and benefit from:

- fast, convenient online submission

- thorough peer review by experienced researchers in your field

- rapid publication on acceptance

- support for research data, including large and complex data types

- gold Open Access which fosters wider collaboration and increased citations

- maximum visibility for your research: over $100 \mathrm{M}$ website views per year
}

At BMC, research is always in progress.

Learn more biomedcentral.com/submissions 